\title{
Forward problem study of an effective medium model for ultrasound blood characterization
}

\author{
Emilie Franceschini, Bloen Metzger, and Guy Cloutier,
}

\begin{abstract}
The Structure Factor Model (SFM) is a scattering model developed to simulate the backscattering coefficient (BSC) of aggregated red blood cells (RBCs). However, the SFM can hardly be implemented to estimate the structural aggregate parameters in the framework of an inverse problem formulation. A scattering model called the Effective Medium Theory combined with the SFM (EMTSFM) is thus proposed to approximate the SFM. The EMTSFM assumes that aggregates of RBCs can be treated as individual homogeneous scatterers, which have effective properties determined by the acoustical characteristics and concentration of RBCs within aggregates. The EMTSFM parameterizes the BSC by three indices: the aggregate radius, the concentration of RBCs within aggregates (also named aggregate compactness) and the systemic hematocrit. The goodness of the approximation of the EMTSFM in comparison with the SFM was then examined. Based on a two-dimensional study, the EMTSFM was found to approximate the SFM with relative errors less than $30 \%$ for a product of the wavenumber times the mean aggregate radius $k r_{a g} \leq 1.32$. The main contribution of this work is the parameterization of the BSC with the RBC aggregate compactness, which is of relevance in clinical hemorheology since it reflects the binding energy between RBCs.
\end{abstract}

E. Franceschini is with the Laboratoire de Mécanique et d'Acoustique LMA - CNRS UPR 7051, 31 chemin Joseph Aiguier, 13402 Marseille cedex 20, France (e-mail: franceschini@1ma.cnrs-mrs.fr).

B. Metzger is with the CNRS - Institut Universitaire des Systèmes Thermiques Industriels IUSTI, Technopôle de Château-Gombert, 5 rue Enrico Fermi, 13453 Marseille cedex 13, France.

G. Cloutier is with the Laboratory of Biorheology and Medical Ultrasonics, University of Montreal Hospital Research Centre (CRCHUM), 2099 Alexandre de Sève (Room Y-1619), Montreal, Quebec H2L 2W5, Canada and with the Department of Radiology, Radio-Oncology and Nuclear Medicine, and Institute of Biomedical Engineering, University of Montreal, Montreal, Quebec, Canada. 


\section{Forward problem study of an effective medium model for ultrasound blood characterization}

\section{INTRODUCTION}

Ultrasonic tissue characterization techniques using the radio frequency (rf) backscattered signals have received broad interest for the past 25 years. One approach is to use the magnitude and frequency dependence of backscatter echoes to quantify the tissue structures such as the size, acoustic impedance, and concentration of the scatterers. This approach has been successfully used for the characterization of the eye [1], the prostate [2], apoptotic cells [3] and the breast [4]. Blood has also been studied by employing this technique [5] [6]. In the ultrasonic blood characterization field, the objective is to assess the level of red blood cell (RBC) aggregation, which is a surrogate marker of inflammation [7]. It is well known that when RBCs are under low shear rates $\left(<10 \mathrm{~s}^{-1}\right)$, they interact strongly with each other and form complex rouleaux or three-dimensional (3D) structures. When the shear rate increases, these rouleaux or structures desaggregate. The aggregation phenomenon in human blood is normal, however hyperaggregation, an abnormal increase of RBC aggregation, is a pathological state associated with several circulatory diseases such as deep venous thrombosis, atherosclerosis and diabetes mellitus. The ultrasonic blood characterization using ultrasound backscatter technique has the potential to provide a method for the non-invasive estimation of the RBC aggregate size. This quantification would help to elucidate the role of RBC aggregation in the etiology of such diseases.

Ultrasound backscattering by blood is mainly due to RBCs that constitute the vast majority (97\%) of the blood cellular content. Blood can thus be described as a biphasic fluid composed of RBCs immersed in plasma. Since RBCs are acoustically considered as weak scatterers (impedance contrast between RBCs and plasma being around $13 \%$ ), multiple scattering can be neglected. However, for such tissue, it is not straightforward to develop a theoretical scattering model because of the high density of RBCs (their volume fraction or hematocrit varies between 30 and $50 \%$ ) and their ability to form aggregates. Theoretical efforts have been made to take into account the high density of RBCs [5] [8]-[10]. In the Rayleigh scattering regime (i.e., for a product of the wavenumber times the scatterer radius $k a \ll 1$ ), Twersky [9] proposed an expression of the backscattered intensity in terms of the single-particle backscattering cross section, number density of particles and packing factor. The packing factor is dependent on the hematocrit but independent on the frequency. This model succeeded to explain the nonlinear relationship between the backscatter amplitude and hematocrit for non-aggregating RBCs [11] but failed to predict the magnitude and frequency dependence of backscatter echoes observed in in vitro experiments when considering aggregating RBCs. That is why Savery and Cloutier [12] proposed to generalize the packing factor theory for aggregating RBCs at a low hematocrit by introducing the frequency dependent structure factor, named the structure factor model (SFM). This model was later generalized to a normal hematocrit of $40 \%$ [13]. The SFM sums the contributions 
from individual RBCs and models the RBC interaction by a statistical mechanics structure factor, which is by definition the Fourier transform of the spatial distribution of RBCs [12]-[14]. Note that the low frequency limit of the structure factor is by definition the packing factor used under Rayleigh conditions, and that the structure factor cannot analytically be calculated contrary to the packing factor [9]. The SFM was largely used to perform simulation studies on RBC aggregation [12], [13], [15]-[17]. Simulations enabled to predict the frequency dependence of the backscattering coefficient (BSC) from various RBC spatial distributions and thus helped the interpretation of experimental observations. However, the SFM cannot directly be used to estimate the RBC aggregate size in the framework of an inverse problem approach because of the intensive computational time to assess the structure factor by realizing distributions of aggregating RBCs with simulations. Please note that the SFM should not be confused with the structure factor size estimator (SFSE) recently proposed by Yu et al. [18] [19]. The SFSE approximates the SFM for practical assessments of RBC structural features (i.e., in an inverse problem formulation).

In details, Yu et al. [18] [19] developed the SFSE scattering theory that approximates the SFM by using a second-order Taylor expansion of the structure factor. The SFSE is thus not as accurate as the SFM. The SFSE parameterizes the BSC by two structure indices: the packing factor and the mean aggregate diameter assumed to be isotropic. However, experiments with pig blood in controlled flow devices [18] [19] and three-dimensional (3D) numerical simulations of isotropic aggregates [16] showed that the two indices are correlated and follow a quadratic relationship under the assumption of isotropic monodisperse aggregates, thus reducing the BSC parameterization to one structural index. Moreover, the SFSE model did not produce good fits to the simulated BSCs computed with the SFM (see Fig. 4 in Ref. [16]). It means that the SFSE model is not sufficient to approximate the SFM accurately; even if the relation between the simulated aggregate sizes and its SFSE parameterization followed a proportional relationship [16]. The goal of this paper was then to propose a new scattering model that better approximates the SFM for structural RBC aggregate characterization.

The scattering theory we propose is based on an Effective Medium Theory (EMT) combined with the SFM, labeled EMTSFM. The EMT was initially developed by Kuster and Toksoz [20] in the field of Geophysics. Herein, the EMT assumes that aggregates of RBCs can be treated as individual homogeneous scatterers, which have effective properties determined by the concentration of RBCs within aggregates and acoustical properties of blood constituents. The approximation of RBC aggregates as homogeneous effective particles is combined with the SFM to consider the concentrated blood medium. The effective particle interactions were thus modeled by a structure factor, as in [12] [13]. The EMTSFM parameterizes the BSC by three indices: the aggregate radius, the internal hematocrit (i.e. the concentration of RBCs within aggregates, also named aggregate compactness) and the systemic hematocrit. Note that the new EMTSFM model would allow characterizing for the first time in the quantitative ultrasound field, the compactness of RBC aggregates (or of any other cellular structures). In the field of clinical hemorheology, assessing the compactness of RBC aggregates is of high clinical importance since it is related to the binding energy between cells. Normal RBC aggregates form rouleaux type structures, whereas pathologies associated with stronger binding energies result in clumps of RBCs (close to a spherical isotropic packing).

The purpose of this study was to compare the EMTSFM and SFM in the framework of a forward problem 
study, i.e. determining the BSC from a known distributions of RBCs with known acoustical parameters with both EMTSFM and SFM. The goodness of the approximation of the EMTSFM in comparison with the SFM was examined as a function of the frequency and of the structural aggregate parameters (i.e., the aggregate size and the internal hematocrit). Because of the computational load to generate $3 \mathrm{D}$ RBC distributions with various internal hematocrits with the SFM, bidimensional (2D) models were used for the BSC computation. Although limitative, this choice allowed us (1) to simulate randomly various internal hematocrits for a given range of systemic hematocrits, and (2) to isolate the effects of aggregate size and internal hematocrit on the BSC.

\section{ULTRASOUND BACKSCATTERING THEORY}

In the following, it is assumed that the incident wavelength $\lambda$ is large compared to the RBC size. Consequently, the RBC shape could be approximated by a simple geometry having an equivalent volume of a RBC in $3 \mathrm{D}$ or having an equivalent surface in 2D [21]. In the present 2D study, RBCs were modeled as parallel infinite cylinders of radius $a$, that have small contrast in acoustical properties relatively to the plasma (see Table I). Two scattering models of RBC aggregation are presented in this section: the SFM and the new EMTSFM.

\section{A. The structure factor model (SFM)}

The SFM of ultrasound backscattering by blood consists of summing contributions from individual RBCs and modeling the RBC interaction by a statistical mechanics structure factor [12] [13] [15]. By considering a collection of $N$ identical and weak scattering RBCs, the BSC expression can be written as:

$$
B S C(-2 \mathbf{k})=m \sigma_{b}(-2 \mathbf{k}) S(-2 \mathbf{k})
$$

where $\mathbf{k}$ is the incident wave vector and $m$ the number density of RBCs that is related to the systemic hematocrit $\phi$ as $m=\phi / A_{p}$ (where $A_{p}$ is the RBC area). The backscattering cross section $\sigma_{b}$ of a single weak RBC can be written as the product of the backscattering cross section of a single RBC in the Rayleigh limit (see Eq. (16) in Ref [9]) and the backscatter form factor $F$ as follows:

$$
\sigma_{b}(-2 \mathbf{k})=\frac{k^{3} A_{p}^{2} \gamma_{z}^{2}}{2 \pi} F(-2 k, a)=\frac{k^{3} A_{p}^{2} \gamma_{z}^{2}}{2 \pi}\left(\frac{J_{1}(2 k a)}{k a}\right)^{2},
$$

where $J_{1}$ is the first order Bessel function of the first kind and $\gamma_{z}=\left(Z_{R B C}-Z_{\text {plasma }}\right) / Z_{\text {plasma }}$ is the fractional variation of impedance between the $\mathrm{RBC}$ and its suspending medium (i.e. the plasma). The form factor $F$ serves as a useful descriptor of scatterer with simple shape [21] [22] and characterizes here the cylindrical shape and size of the 2D RBC scatterer. The demonstration of the cylindrical form factor expression in $2 \mathrm{D}$ is given in appendix. The function $S$ in Eq. (1) is the structure factor representing the spatial positioning of RBCs and is defined by:

$$
S(-2 \mathbf{k})=E\left[\frac{1}{N}\left|\sum_{i=1}^{N} e^{-i 2 \mathbf{k r}_{i}}\right|^{2}\right]
$$

where $E$ represents the expected value of a random variable and $\mathbf{r}_{i}$ the position vectors defining the center of the $i$ th $\mathrm{RBC}$ in space. Note that the aggregation phenomenon is only affecting the structure factor since RBC properties 
(i.e. $\left.\sigma_{b}\right)$ and the systemic hematocrit are expected to remain constant in the modeled region of interest.

In the case of disaggregated RBCs, the low frequency limit of the structure factor is a constant with this model $(S(k) \rightarrow S(0)=W)$, which is the packing factor. The BSC expression can thus be simplified as follows:

$$
B S C_{L F}(-2 \mathbf{k})=m W \sigma_{b}(-2 \mathbf{k})
$$

The most often used packing factor expression is based on the Percus-Yevick pair-correlation function for identical, hard and radially symmetric particles. The Percus-Yevick packing factor $W_{P Y}$ was first applied to blood by Shung and coworkers, and it is related to the systemic hematocrit in the 2D space as [11]

$$
W_{P Y}=\frac{(1-\phi)^{3}}{1+\phi}
$$

The packing factor reflects the positional correlation amongst particles: $W=1$ for completely random distributions and $W$ tends toward zero when the particle number as well as the spatial correlation between particles increase.

\section{B. The proposed model: an effective medium theory combined with the SFM (EMTSFM)}

As a first approximation, we assume that all the RBCs are aggregated in blood, that the aggregates are identical and isotropic and that the RBCs within the aggregates are evenly distributed. The EMTSFM assumes that aggregates of RBCs can be treated as individual homogeneous scatterers as shown in Fig. 1. Each aggregate is thus approximated by an effective single particle (i.e. in this $2 \mathrm{D}$ study, an effective single cylinder) having a radius $r_{a g}$. The density $\rho_{a g}$ and compressibility $\kappa_{a g}$ of the new effective particle are determined by considering the EMT [20]. It means that $\rho_{a g}$ and $\kappa_{a g}$ are derived from the acoustical properties of the two fluids that constitute the aggregates (i.e. $\rho_{1}$, $\rho_{2}, \kappa_{1}$ and $\kappa_{2}$, where 1 indicates properties of RBCs and 2 those of plasma) and from the internal concentration of RBCs within the aggregates, defined as the internal hematocrit $\phi_{i}$, as follows:

$$
\begin{aligned}
\rho_{a g} & =\phi_{i} \rho_{1}+\left(1-\phi_{i}\right) \rho_{2} \\
\frac{1}{\kappa_{a g}} & =\frac{\phi_{i}}{\kappa_{1}}+\frac{1-\phi_{i}}{\kappa_{2}}
\end{aligned}
$$

The acoustic interaction of RBCs within aggregates is therefore taken into account with the EMT, similarly to the SFM where it is considered by the structure factor that models the individual position of RBCs and their acoustical interaction whether they are within an aggregate or not. The main advantage of the EMTSFM is the consideration of the compactness of aggregates with the parameter $\phi_{i}$.

The BSC from blood is then obtained by summing contributions from individual effective particles of radius $r_{a g}$ and modeling the effective particle interaction by a statistical mechanics structure factor $S_{a g}$. The equivalent BSC expression is thus given by:

$$
B S C_{e q}(-2 \mathbf{k})=m_{a g} \sigma_{a g}(-2 \mathbf{k}) S_{a g}(-2 \mathbf{k})
$$

where $m_{a g}$ is the number density of aggregates that is related to the effective area fraction of aggregates $\phi_{a g}$. The effective area fraction of aggregates is equal to the area fraction of RBCs in blood $\phi$ divided by the internal 
hematocrit $\phi_{i}: \phi_{a g}=\phi / \phi_{i}$. The backscattering cross section $\sigma_{a g}$ of an effective single cylinder was calculated using the fluid-filled cylinder model developed by Doolittle and Uberall [23]. That model provides an exact solution for the backscattering of sound by a single fluid cylinder, not necessarily small compared to the wavelength, in a surrounding fluid medium (i.e. the plasma). In the proposed forward problem study, the radius $r_{a g}$, density $\rho_{a g}$ and compressibility $\kappa_{a g}$ of the aggregates as well as the density $\rho_{2}$ and compressibility $\kappa_{2}$ of the plasma are known a priori (see Table I) such that the backscattered pressure of the effective fluid cylinder could be computed as a function of frequency. The structure factor $S_{a g}$ was calculated for a collection of $N_{a g}$ identical and disaggregated particles (mimicking RBC aggregates) of radius $r_{a g}$ randomly distributed as follows:

$$
S_{a g}(-2 \mathbf{k})=E\left[\frac{1}{N_{a g}}\left|\sum_{i=1}^{N_{a g}} e^{-i 2 \mathbf{k r}^{\prime}{ }_{i}}\right|^{2}\right]
$$

where $\mathbf{r}_{i}$ are the position vectors defining the center of the $i$ th effective particle (or aggregate) in space. Note that in the research field of optics [24], the applicability of the modeling approach based on the structure factor (called the interference approximation) is limited to a product $k R \leq 3.5$ (or $k R \leq 1.5$, respectively) for a relative refractive index of the particles equal to 1.19 (or 1.8), with $R$ the radius of isotropic identical particles. Since the relative acoustic impedance of RBCs $z_{1} / z_{2}$ is around 1.12 (see Table I), we are thus expecting the same limitation for the EMTSFM (i.e., a validity of this modeling approach restricted to $k r_{a g} \leq 3.5$ ).

In the low frequency limit, for aggregate sizes small compared to the wavelength, the structure factor $S_{a g}$ can be well approximated by the Percus-Yevick packing factor of Eq. (5) for effective particles. The equivalent BSC expression is thus simplified as follows:

$$
B S C_{e q_{L F}}(-2 \mathbf{k})=m_{a g} \frac{\left(1-\phi_{a g}\right)^{3}}{1+\phi_{a g}} \sigma_{a g}(-2 \mathbf{k}),
$$

where $\sigma_{a g}$ is calculated using the exact model of fluid-filled cylinders [23], such that, in comparison with Eq. (7), the only approximation is the structure factor $S_{a g}$.

\section{Simulation MEthods}

The computation of the $B S C$ and $B S C_{e q}$ requires the knowledge of the structure factors $S$ and $S_{a g}$ as described in Eq. (1) and Eq. (7). Since the structure factor $S$ (or equivalently $S_{a g}$ ) is by definition a statistical quantity, an average of all structure factors obtained from several particle distributions can give an estimated value of $S$ (or equivalently $S_{a g}$ ). Note that 2D models were used because of the computational time to generate 3D RBC distributions with the SFM. Although limitative, this choice allowed us to simulate randomly various internal hematocrits for a given range of systemic hematocrits.

\section{A. Computation of particle distributions}

1) Distribution of RBCs for the computation of $S$ with the SFM: We describe here how random distributions for non-aggregating and aggregating RBCs were computed within the simulated surface area $L^{2}$ of $600^{2} \mu m^{2}$. The RBC particle radius $a$ was set to $2.75 \mu \mathrm{m}$ for all simulations. In the case of disaggregated RBCs, particles 
were randomly distributed (using a random number generator) with non-overlapping positions to give the desired area fraction of RBCs in blood (i.e. the systemic hematocrit $\phi$ ). In the case of aggregated RBCs, we first specified the aggregate radius and then aggregates were randomly distributed with non-overlapping positions to give the desired area fraction of aggregates $\phi_{a g}$. RBC distributions within aggregates were then generated by considering the number of RBCs within aggregates $n_{i}$ and the area fraction of RBCs within aggregates $\phi_{i}$. It is important to emphasize that random particle distributions could be easily generated using a random number generator up to an area fraction of approximatively 0.5 . In our study, the area fraction of RBCs within aggregates $\phi_{i}$ could be enlarged, up to a maximum value $\phi_{i_{\max }}$ fixed to 0.6 . The procedure we chose to reach such a high number of RBCs within aggregates is described in the following.

We first randomly distributed $n_{i}$ RBCs on a total area that was larger than the aggregate area, such that the initial area fraction of RBCs within aggregates was lower than 0.5. Then, in order to increase this area fraction, the RBCs were submitted to an external force oriented toward the center of mass of the RBC spatial distribution. RBCs moved toward each other while a repulsive force prevented them from overlapping. These forces were maintained until the area fraction of RBCs inside the considered aggregate reached the desired area fraction $\phi_{i}$. This distribution procedure was repeated for each aggregate such that the distribution of RBCs within each aggregate was different. Note that this procedure is not time consuming if a small number of RBCs is treated: herein the maximum number of RBCs within aggregates was equal to 38 for the maximum aggregate radius of $7.95 a=21.86 \mu \mathrm{m}$. The maximum value of aggregate area fraction $\phi_{a g_{\max }}$ was thus fixed to 0.5 and as a consequence, the maximum value of the systemic hematocrit $\phi_{\max }$ was limited to: $\phi_{\max }=\phi_{a g_{\max }} \phi_{i_{\max }}=0.3$.

Figure 2 illustrates spatial arrangements of RBCs for three internal hematocrits of 40, 50 and $60 \%$. In these simulations, the aggregate radius had a constant value of $r_{a g} / a=6.32$ (i.e. $r_{a g}=17.39 \mu \mathrm{m}$ ) and a constant systemic hematocrit of $20 \%$.

2) Distribution of effective particles for the computation of $S_{a g}$ with the EMTSFM: Once the effective particle radius was specified, particles were randomly distributed using a random number generator with non-overlapping positions to give the desired area fraction $\phi_{a g}$. Several distributions were performed with different effective particle radii varying from $3.16 a(=8.69 \mu \mathrm{m})$ to $7.95 a(=21.86 \mu \mathrm{m})$ corresponding to different studied aggregate sizes.

\section{B. BSC computation}

For each distribution of RBCs (or effective particles, respectively), density matrices $D$ (or $D_{a g}$ ) were computed by dividing the square simulation plane $L^{2}$ in $N_{p}^{2}$ pixels (herein, $N_{p}=512$ ) and by counting the number of RBCs (or the number of effective particles) falling into each pixel. These matrices represented samplings of microscopic density functions defined by

$$
\begin{aligned}
& D(\mathbf{r})=\sum_{i=1}^{N} \delta\left(\mathbf{r}-\mathbf{r}_{i}\right), \text { for the } B S C \text { computation with the SFM } \\
& D_{a g}\left(\mathbf{r}^{\prime}\right)=\sum_{i=1}^{N_{a g}} \delta\left(\mathbf{r}^{\prime}-\mathbf{r}_{i}^{\prime}\right), \text { for the } B S C_{e q} \text { computation with the proposed EMTSFM }
\end{aligned}
$$


where $N$ is the number of RBCs in blood, $N_{a g}$ the number of aggregates and $\delta$ the Dirac distribution. According to equations (3) and (8) and as explicitly described in Appendix of [14], the structure factors can thus be described by:

$$
\begin{aligned}
& S(-2 \mathbf{k})=E\left[\frac{1}{N}\left|\int D(\mathbf{r}) e^{-i 2 \mathbf{k r}} d \mathbf{r}\right|^{2}\right] \text { and } \\
& S_{a g}(-2 \mathbf{k})=E\left[\frac{1}{N_{a g}}\left|\int D_{a g}\left(\mathbf{r}^{\prime}\right) e^{-i 2 \mathbf{k r}^{\prime}} d \mathbf{r}^{\prime}\right|^{2}\right] .
\end{aligned}
$$

Each structure factor was thus computed by averaging 2-D fast Fourier transforms (2D FFT) of 400 density matrices for averaging purpose. The FFTs gave the structure factor values $S(-2 \mathbf{k})$ and $S_{a g}(-2 \mathbf{k})$ on a centered grid of wavevectors between $\pm \pi N_{p} / 2 L$ with a step of $\Delta k=\pi / L$. For the SFM, the $B S C$ was thus obtained using Eq. (1). For the EMTSFM, the $B S C_{e q}$ was computed using Eq. (7).

\section{RESULTS}

In the following, the relative errors for $B S C_{e q}$ computed with the new EMTSFM were evaluated as $\left(B S C_{e q^{-}}\right.$ $B S C) / B S C_{e q}$, where the $B S C$ of the SFM is assumed as the exact solution.

\section{A. Influence of the internal hematocrit}

A key feature of the new simulation method was the possibility to simulate randomly various internal hematocrits corresponding to different compactness of aggregates. Frequency-dependent backscattering coefficients with different internal hematocrits from 30 to $60 \%$ are given in Fig. 3(a) and (b) at systemic hematocrits of 10 and $20 \%$ over a large frequency range (4 MHz - $100 \mathrm{MHz}$ ). Corresponding relative errors for the $B S C_{e q}$ are given in Fig. 3(c) and (d). It was assumed that the aggregates had a constant radius of $r_{a g} / a=6.32$ (i.e. $r_{a g}=17.39 \mu m$ ). Note that for the

$20 \%$ systemic hematocrit, internal hematocrits smaller than $40 \%$ could not be computed because the corresponding area fractions of aggregates were too high: $\phi_{a g}>0.5$. As seen on figures 3(a) and (b), as the internal hematocrit $\phi_{i}$ increases, the $B S C$ amplitude increases at low frequencies $(<23 \mathrm{MHz})$ and decreases at high frequencies $(>28$ MHz). The first peaks of the $B S C$ are between 18.0 and $20.6 \mathrm{MHz}$ for all simulated conditions. The relative error for $B S C_{e q}$ was less than $30 \%$ for frequencies below $18 \mathrm{MHz}$ (see figures 3(c) and (d)). This $18 \mathrm{MHz}$ frequency corresponds to a product $k r_{a g}=1.27$. More generally, at frequencies less than $23 \mathrm{MHz}$, backscattering coefficients obtained with the SFM and the EMTSFM have the same behaviors: the first peaks of $B S C$ and $B S C_{e q}$ occur at the same frequencies and the magnitude of both $B S C$ and $B S C_{e q}$ increase when the internal hematocrit becomes higher.

A quantitative ultrasonic parameter that has often been used for tissue characterization is the spectral slope (SS). The SS is the linear slope of the BSC as a function of frequency on a log-log scale. The variation of SS with the internal hematocrit is given in Fig. 4 for systemic hematocrits of 10 and 20\%. The solid lines represent the SSs for the $B S C$ computed with the SFM and the dashed lines the SSs for the $B S C_{e q}$ computed with the EMTSFM. For both SFM and EMTSFM, the SS behavior versus the internal hematocrit is the same: the SS decreases with the increase of the internal hematocrit, except for the configuration $\phi=20 \%, \phi_{i}=40 \%$. Note also that the SS of the EMTSFM slightly underestimated the SS of the SFM with a maximum difference of 0.23. 


\section{B. Influence of the aggregate size}

Figure 5 shows backscattering coefficients as a function of frequency for different aggregate sizes and for systemic hematocrits of 10, 20 and $30 \%$. In these simulations, the internal hematocrit was the highest: $\phi_{i}=60 \%$. The symbols represent the $B S C$ computation for the disaggregated case $\left(r_{a g} / a=1\right)$ and for aggregated cases with radii of $r_{a g} / a=3.16,5.0$ and 7.07. Also given are the corresponding $B S C_{e q}$ computed with the EMTSFM (see Eq. (7)) in dashed lines. For frequencies less than $20 \mathrm{MHz}$, the amplitudes of both $B S C$ and $B S C_{e q}$ increase with the size of aggregates. Moreover, the peaks of both $B S C$ and $B S C_{e q}$ occur at lower frequencies as the aggregate radius increases.

The low frequency approximation of the EMTSFM (i.e. using the Percus-Yevick packing factor) was also studied for the same configurations. The $B S C_{e q_{L F}}$ computed by Eq. (9) are given in Figure 6, along with plots of the $B S C$ computed with the SFM. As expected, the error for $B S C_{e q_{L F}}$ is larger than the error for $B S C_{e q}$ at high frequencies. The error between $B S C_{e q_{L F}}$ and $B S C$ becomes larger as the systemic hematocrit $\phi$ increases. Nevertheless, it is interesting to observe that at low frequencies less than $5 \mathrm{MHz}$, the amplitude of the $B S C_{e q_{L F}}$ matches very well the exact $B S C$, as expected.

For systemic hematocrits of 10,20 and $30 \%$, the relative errors for $B S C_{e q}$ and $B S C_{e q_{L F}}$ were studied for a constant value of the internal hematocrit $\phi_{i}=60 \%$ and for eleven sizes $r_{a g} / a$ varying from 3.16 to 7.95 with a step of around 0.5. For each case, the frequency limit $f_{l}$ and the corresponding product $k_{l} r_{a g}$ for which the relative error was less than 30\% were determined. Results are summarized in Fig. 7. The solid lines are for the EMTSFM and the dashed lines for the low frequency approximation of the EMTSFM using the Percus-Yevick packing factor. It reveals that the EMTSFM gives accurate estimates of BSC until $k r_{a g} \leq 1.32$ on average (i.e. for all the tested values of $r_{a g} / a$ ), whereas the EMTSFM at low frequency is only accurate until approximately $k r_{a g} \leq 0.73$.

Figure 8 shows the influence of the aggregate size on the SS for different systemic hematocrits. The solid lines represent the SSs for the $B S C$ computed with the SFM and the dashed lines the SSs for the $B S C_{e q}$ computed with the EMTSFM. The results of SS presented in Figure 8 showed that the backscattered power by disaggregated RBCs $\left(r_{a g} / a=1\right)$ increased with the third power of wave frequency (SS=3), as it was expected by the Rayleigh theory in 2D. The SS behavior with the aggregate size is the same for both SFM and EMTSFM: as the aggregate size increases, the SS decreases at $\phi=10 \%$, whereas the SS increases at $\phi=20 \%$ and $30 \%$. The maximum differences between slopes of the EMTSFM and slopes of the SFM is around 0.35 for all studied hematocrits (i.e. relative error for the SS of $B S C_{e q}$ around 9\%) and it corresponds to the largest aggregate size.

\section{DISCUSSION}

\section{A. Limitation of the EMTSFM with respect to $k r_{a g}$}

Based on the new EMTSFM theory, the BSC from aggregated RBCs was parameterized by three indices: the aggregate size, internal hematocrit and systemic hematocrit. The aim of our study was to estimate the goodness of the approximation of the EMTSFM in comparison with the SFM. We found that the EMTSFM had a relative error less than $30 \%$ for a mean product $k r_{a g} \leq 1.32$ at all internal hematocrits and systemic hematocrits studied. 
Although the EMTSFM gave estimates with large errors for $k r_{a g}>1.32$, the EMTSFM fitted very well the BSC curve computed with the SFM for all tested factors (i.e. $r_{a g}, \phi_{i}$ and $\phi$ ) in the frequency bandwidth between 4 $\mathrm{MHz}$ and the frequency position of the first peak. This result is qualitatively consistent with works in optics [24] where the limits of applicability of the interference approximation based on the structure factor were found for a volume fraction of particles smaller than $10 \%$ and for a product $k R \leq 3.5$ (or $k R \leq 1.5$, respectively) when the relative refractive index of the particles is 1.19 (or 1.8). These limits were determined by comparing theoretical predictions of the interference approximation with theoretical predictions of a much more exact quasi-crystalline approximation and with experimental data of a polystyrene latex suspension in water. In comparison with our results in acoustics where the relative acoustic impedance of the RBCs is around 1.12, the limit of applicability of the proposed EMTSFM in terms of particle size is surprisingly close to the optic limit $k R \leq 1.5$, when one considers a large relative refractive index of 1.8. In terms of particle concentration, the EMTSFM showed no limitation with respect to the studied hematocrit (up to $30 \%$ ), contrary to the interference approximation valid up to a volume fraction of $10 \%$.

\section{B. Variation of the spectral slope with $\phi_{i}$ and with $r_{a g}$}

On the goodness of the EMTSFM modeling the SS, notice that the variations of SS with $\phi_{i}$ and $r_{a g}$ followed similar trends for both SFM and EMTSFM (figures 4 and 8). The SS of the EMTSFM slightly underestimated the SS of the SFM with a maximum relative error of $9 \%$. As it can be observed in Fig. 8, as the aggregate size increased, the difference between slopes of the EMTSFM and SFM increased. It is due to the constant frequency bandwidth chosen between 5 and $15 \mathrm{MHz}$ for all studied aggregate sizes. Indeed, in the frequency bandwidth between $4 \mathrm{MHz}$ and the frequency position of the first peak, the relative error for the $B S C_{e q}$ increased with the frequency as it was shown in figures 3(c) and (d). Since the relative error was less than $30 \%$ for a mean product $k r_{a g} \leq 1.32$, the mean relative error in the frequency bandwidth 5-15 MHz for the smallest aggregates was smaller that the relative error for the largest aggregates in the same frequency bandwidth.

Contrary to blood modeling, the ultrasound characterization of tissues such as the breast [4] or the liver [25] assumes scatterers to be randomly distributed at a low number density. Tissue models are generally based on 3D spatial autocorrelation functions describing the shape and isotropic random distribution of scatterers in the medium, and modeling of the spatial autocorrelation function could be gaussian, spherical or exponential [22] [25]. Based on these models, the SS is an indicator of the scatterer size, and a decrease in slope usually corresponds to an increase in scatterer size [25]. That is why it is interesting to discuss the variation of the SS with the aggregate size in the framework of the SFM or EMTSFM that considers the structure factor (see Fig. 8). For the lowest systemic hematocrit of $10 \%$, the SS decreased with the increase in aggregate size. Since the hematocrit is low, the known behavior of the SS was observed. However, the SS increased with the aggregate size for systemic hematocrits of 20 and $30 \%$. The structure factor, which models the spectral behavior of the scatterer spatial distributions, caused this behavior of the SS. Indeed, according to Eq. (1) (or Eq. (7), respectively), the BSC frequency dependence (or the $B S C_{e q}$ frequency dependence) is determined by the frequency dependences of $\sigma_{b}$ and $S$ (or $\sigma_{a g}$ and $S_{a g}$ ). 
To illustrate this, the structure factors $S$ and $S_{a g}$ corresponding, respectively, to the SFM and the EMTSFM are displayed in Fig. 9 for a fixed systemic hematocrit $\phi=30 \%$, a fixed internal hematocrit $\phi_{i}=60 \%$ and three aggregate radii $r_{a g} / a=3.16,5.0$ and 7.07. The plot of $S$ is represented in a larger frequency bandwidth up to $320 \mathrm{MHz}$ in order to show the high-frequency oscillations of the structure factor around 1. In the frequency bandwidth 5-15 $\mathrm{MHz}$, the frequency dependence of the structure factors $S$ and $S_{a g}$ do not follow a simple power law, since the structure factors $S$ and $S_{a g}$ versus frequency on a log-log scale are non-linear curves. One could observe that, in the frequency bandwidth 5-15 MHz, both the structure factors $S$ and $S_{a g}$ increase with the frequency, and the larger is the aggregate size the larger is the increase. That is why the SS for both $B S C$ and $B S C_{e q}$ increased above 3 with the aggregate size for this studied systemic hematocrit of 30\%. The increase of SS with the aggregate size we observed in the current study is consistent with an earlier 2D numerical simulation study performed by Fontaine and Cloutier [17]. In this 2D study, the SS between 5-25 MHz could increase from 3.1 to 3.3 when the level of aggregation increased for an hematocrit of 40\%. Moreover, in vitro Couette flow experiments performed in [18] showed the SS superior to 4 between 9 and $15 \mathrm{MHz}$ for shear rates of 2 and $10 \mathrm{~s}^{-1}$ at a systemic hematocrit of $40 \%$ (see figure $4 b$ in Ref [18]).

It is important to notice that we found that the SS decreased as $\phi_{i}$ increased for all studied systemic hematocrits. On the other hand, the behavior of the SS as a function of the aggregate size depended on the systemic hematocrit. As a consequence, the parameter SS seems well adapted to observe a change in the internal hematocrit. But in experimental conditions where effects of aggregate size and internal hematocrit are mixed, the SS cannot be a useful index for blood characterization.

\section{Computation of RBC distributions}

The method we proposed here to obtain the RBC spatial distribution did not take into consideration realistic interactions between RBCs. It was a simple and fast method to generate samples containing non-overlapping, identical and isotropic aggregates. The main advantage of this method was the possibility to have various internal hematocrits with the same size of aggregates. The simulation results showed that the frequency position of the BSC first peak was very little affected by changes in the internal hematocrit whereas it was greatly affected by changes in the aggregate size. Indeed, the change in frequency position was only $1.5 \mathrm{MHz}$ (and respectively $2.1 \mathrm{MHz}$ ) for the hematocrit of $10 \%$ (and 20\%) when the internal hematocrit increased from 30 to $60 \%$. Moreover, theoretical predictions of the BSC with the SFM showed that its amplitude increased as $r_{a g}$ and $\phi_{i}$ increase (see figures 3 and 5). To our knowledge, the influence of the internal hematocrit on the BSC was not studied previously, mainly because of simulation methods used to realize distributions of aggregating RBCs. These methods were based on particle dynamics or statistical mechanics [12] [13] [15]-[17] and had the objective to mimic the rheological behavior of blood. The RBC distributions obtained showed aggregates with anisotropic shape [12] [13] [15] [17] [26] and/or a polydispersity in terms of aggregate sizes, aggregate shapes and/or internal hematocrits [12] [13] [15]-[17]. As mentioned earlier, contrary to those earlier studies, the 2D simulation method we proposed for distributing RBCs allowed isolation of the effects of the aggregate size and internal hematocrit, and to better understand the role of 
each parameter. In that sense, our study has provided some insights into the influence of the aggregate size and internal hematocrit on the BSC frequency dependence. The internal hematocrit as well as the aggregate size can greatly influence the BSC amplitude. The frequency position of the BSC first peak was found not to be significantly affected by changes in the internal hematocrit whereas it was greatly affected by changes in the aggregate size.

\section{On the use of the EMTSFM in vivo}

Although the present work allowed a fundamental study on the influences of the internal hematocrit and aggregate size on the BSC, the method we chose to distribute particles was limited to area fractions of aggregates $\phi_{a g} \leq 0.5$, area fraction of RBCs within aggregates $\phi_{i} \leq 0.6$ and systemic hematocrits $\phi \leq 0.3$. One might thus question the use of the EMTSFM for in vivo experiments with a physiological hematocrit $\phi$ typically varying between 0.3 and 0.5. It is important to recall that the only intensive computation with the proposed EMTSFM is the structure factor $S_{a g}$ needing the computation of effective particle distributions. If one considers the application of the EMTSFM in vivo, 3D effective particle distributions must be considered as well as the range of typical volume fractions. For example, the volume fraction of RBCs within aggregates $\phi_{i}$ may physiologically be larger than $60 \%$ since RBCs are deformable. By considering a systemic hematocrit of $40 \%$ (and 20\%, respectively) and extrema values of $\phi_{i}$ between 60 and 90\%, the volume fraction of aggregates $\phi_{a g}$ would then vary between 44 and $66 \%$ (between 22 and 33\%). For future simulations, particle distributions in 3D could be easily generated using a random number generator up to a volume fraction of approximatively 0.3. It means that the method used to compute the $B S C_{e q}$ in this paper may easily be applied in 3D up to a volume fraction $\phi$ of $20 \%$. For such low volume fractions of cells, one could envisage to use the EMTSFM for other biomedical applications such as cancer [4] [27], where the cells can be locally densely packed (see for example Figure 5 in [4]). In the case of blood applications, in order to deal with a physiological hematocrit of $40 \%$ and a maximum volume fraction of aggregates of $66 \%$, one may envisage distributing particles in a random close packing configuration where the attainable volume fraction could be up to $64 \%[28]$.

On the use of the EMTSFM in vivo, one can also question the practical value of that model assuming isotropic aggregates. In human blood, low shear rates can promote the formation of RBC aggregates having anisotropic (i.e. rouleaux) or isotropic (i.e. clump) structures. The rouleaux like pattern is typically associated to normal blood. However, as the binding energy between RBCs increases with inflammation [29], aggregates form clump structures such as in diabetes mellitus [30] [31]. The assumption of isotropic aggregates in the EMTSFM is thus valid as far as we are concerned with the study of pathological states. In the case of normal human rouleaux of RBCs, if the EMTSFM is applied to estimate structural parameters such as the internal hematocrit and the aggregate size, this assumption would obviously create a bias against the parameter estimation.

Another important aspect to consider is the assumption of identical aggregates in the current EMTSFM implementation. Under in vivo conditions with ultrasound measurements on a blood vessel, the shear rate distribution 
varies with the radial position, and consequently, the aggregate size distribution too. That is why the backscattered echoes from blood are generally analyzed over a rectangular or a hamming window which is moved along the RF signal to examine the whole vessel at different depths [19] [32]. For example, at a central frequency of 25 $\mathrm{MHz}$, the window length was typically around $400 \mu m$ [19] [32] [33]. RBC aggregates may thus be assumed to be locally identical. However, one may also expect that the aggregate size varies around a mean value for a given radial position within a blood vessel. An interesting simulation study was recently performed in 2D by Vlad et al. [34]. According to this paper, the increase in particle size variance (or equivalently, in aggregate size variance) is expected to increase the BSC amplitude and not to significantly affect the frequency position of the BSC first peak (see figure 2 in Ref. [34]). One could thus predict the bias against the parameter estimation with the EMTSFM if there is a variance in aggregate sizes. The discussion next considers small and large aggregates with respect to the observation of Vlad et al. [34].

In the case of small aggregates, the BSC first peak occurs at high frequencies and is thus generally not captured in the measured frequency bandwidth of the transducer (typically from $12 \mathrm{MHz}$ to $38 \mathrm{MHz}$ for a $100 \%$ bandwidth at 25 MHz). Readers may refer to Fig. 4b of Ref. [18] and to Fig. 3 of Ref. [19] to see measured BSCs from aggregating porcine RBCs in a controlled Couette flow. Because of the predicted increase in BSC amplitude with the increase in particle size variance [34], the assumption of identical aggregates in the current study may result in overestimations of the aggregate size and/or of the internal hematocrit. On the other hand, in the case of large aggregates, the BSC first peak occurs at low frequencies and would likely be within the measured frequency bandwidth of the transducer. In this case, the assumption of identical aggregates may not greatly affect the aggregate size estimation but may result in an overestimation of the internal hematocrit. The EMTSFM is thus expected to better estimate the size of large aggregates and the estimation of the internal hematocrit may be overestimated in the case of polydisperse aggregates, whatever their dimensions.

\section{CONCLUSION}

The SFM is recognized to be a good model to simulate the BSC from aggregated RBCs. However, it cannot directly be applied to estimate the structural aggregate parameters in the framework of an inverse problem formulation. The EMTSFM was thus proposed to approximate the SFM and the goodness of the EMTSFM in comparison with the SFM was examined. Contrary to the SFM, the EMTSFM can be implemented to estimate structural aggregate parameters.

The EMTSFM parameterizes the BSC by three indices: the aggregate size, the internal hematocrit (or aggregate compactness) and the systemic hematocrit. In comparison with the SFM, the EMTSFM provided accurate quantitative estimates of the BSC for $k r_{a g} \leq 1.32$ for studied conditions. Moreover, even if $k r_{a g}>1.32$, the EMTSFM provided a good approximation of the BSC: indeed, the BSC amplitude was well approximated in the frequency bandwidth between $4 \mathrm{MHz}$ and the frequency position of the first peak, and the high-frequency positions of peaks were well identified.

Another important finding was the variation of the SS with $\phi_{i}$ and $r_{a g}$. The SSs for both $B S C$ and $B S C_{e q}$ 
computed with the SFM and EMTSFM followed the same trends of variations with $\phi_{i}$ and $r_{a g}$. We found that the SS decreased as the internal hematocrit increased for all studied systemic hematocrits. On the other hand, the behavior of the SS as a function of the aggregate size depended on the systemic hematocrit. For the largest hematocrits (20 and $30 \%$ ), the SS increased as the aggregate size increased. Consequently, the SS cannot be a useful index for blood characterization of RBC aggregation.

To conclude, the EMTSFM approximates the SFM satisfactorily for a product $k r_{a g} \leq 1.32$. This suggests that the EMTSFM is an adequate model for blood characterization. An important contribution of this new model is the parameterization of the BSC with the aggregate compactness, which is a structural parameter not available in any other modeling strategies proposed in quantitative ultrasound imaging. In future, it would be interesting to study two other important factors: 1) the polydispersity in terms of aggregate sizes and internal hematocrits and 2) the non-sphericity of the aggregates. Another important study would be the estimation of structural aggregate parameters with the EMTSFM that could be compared to existing methods such as the SFSE [16] [18] [19] and the traditional gaussian model developed by Lizzi [6] [25].

\section{APPENDIX}

The form factor is generally given in 3D [21] [22]. That is why the demonstration to obtain the 2D form factor of a fluid infinite cylinder of radius $a$ is given here. In the Born approximation (i.e. for weak scattering conditions), the form factor has a simple expression that is related to the spatial Fourier spectrum of the impedance contrast (see Eq. (5) in Ref [21] for the form factor expression in 3D):

$$
F(-2 k, a)=\frac{1}{A_{p}^{2} \gamma_{z}^{2}}\left|\int_{A_{p}} \gamma_{z}(\mathbf{r}) e^{2 i \mathbf{k r}} d^{2} \mathbf{r}\right|^{2}
$$

We can introduce cylindrical coordinates to obtain the following expression:

$$
\begin{aligned}
F(-2 k, a) & =\frac{1}{A_{p}^{2} \gamma_{z}^{2}}\left|\int_{0}^{a} \int_{-\pi}^{\pi} \gamma_{z} e^{2 i k r \cos (\theta)} r d r d \theta\right|^{2} \\
& =\frac{1}{A_{p}^{2}}\left|\int_{0}^{a} 2 \pi r J_{0}(2 k r) d r\right|^{2} \\
& =\frac{(2 \pi)^{2}}{\left(\pi a^{2}\right)^{2}}\left(\frac{2 k a J_{1}(2 k a)}{(2 k)^{2}}\right)^{2} \\
& =\left(\frac{J_{1}(2 k a)}{k a}\right)^{2}
\end{aligned}
$$

where $J_{0}$ is the Bessel function of the first kind of order 0 .

\section{ACKNOWLEDGMENTS}

This work was supported by the Fonds Incitatifs Recherche 2010 of the Université de Provence and by a grant from the Canadian Institutes of Health Research ( $\sharp$ MOP-84358). The authors would like to thank Prof. Richard E. Challis for his helpful discussion on the effective medium approach. 


\section{REFERENCES}

[1] E. J. Feleppa, F. L. Lizzi, D. J. Coleman, and M. M. Yaremko, "Diagnostic spectrum analysis in ophthalmology: a physical perspective", Ultrasound Med. \& Biol., vol. 12, pp. 623-631, 1986.

[2] E. J. Feleppa, T. Liu, A. Kalisz, M. C. Shao, N. Fleshner, and V. Reuter, "Ultrasonic spectral-parameter imaging of the prostate", Int. J. Imag. Syst. Technol., vol. 8, pp. 11-25, 1997.

[3] M. C. Kolios, G. J. Czarnota, M. Lee, J. W. Hunt, and M. D. Sherar, "Ultrasonic spectral parameter characterization of apoptosis, Ultrasound in Med. \& Biol., vol. 28, pp. 589-597, 2002.

[4] M. L. Oelze and J. F. Zachary, "Examination of cancer in mouse models using high frequency quantitative ultrasound", Ultrasound in Medicine \& Biology, vol. 32, pp. 1639-1648, 2006.

[5] L. Y. L. Mo and R. S. C. Cobbold, "Theoretical models of ultrasonic scattering in blood", in Ultrasonic Scattering in Biological Tissues, edited by K. K. Shung and G. A. Thieme (CRC, Boca Raton, FL, 1993), Chap.5, pp.125-170.

[6] H. Kitamura, B. Sigel, J. Machi, E. J. Feleppa, J. Sokil-Melgar and J. Justin, "Roles of hematocrit and fibrinogen in red cell aggregation determined by ultrasonic scattering properties", Ultrasound Med. \& Biol., vol. 21, pp. 827-832, 1995.

[7] R. B. Ami, G. Barshtein, D. Zeltser, Y. Goldberg, I. Shapira, A. Roth, G. Keren, H. Miller, V. Prochorov, A. Eldor, S. Berliner and S. Yedgar, "Parameters of red blood cell aggregation as correlates of the inflammatory state", Am. J. Physiol. Heart Circ. Physiol., vol. 280, pp. H1982-H1988, 2001.

[8] L. Y. L. Mo and R. S. C. Cobbold, "A stochastic model of the backscattered Doppler ultrasound from blood", IEEE Trans. Biomed. Eng., vol. 33, pp. 20-27, 1986.

[9] V. Twersky, "Low-frequency scattering by correlated distributions of randomly oriented particles", J. Acoust. Soc. Am., vol. 81, no. 5, pp. 1609-1618, 1987.

[10] L. Y. L. Mo and R. S. C. Cobbold, "A unified appraoch to modeling the backscattered Doppler ultrasound from blood", IEEE Trans. Biomed. Eng., vol. 39, pp. 450-461, 1992.

[11] K. K. Shung, "On the ultrasound scattering from blood as a function of hematocrit", IEEE Trans. Sonics Ultrason. SU-29, pp. 327-331, 1982.

[12] D. Savery and G. Cloutier, "A point process approach to assess the frequency dependence of ultrasound backscattering by aggregating red blood cells", J. Acoust. Soc. Am., vol. 110, no. 6, pp. 3252-3262, 2001.

[13] I. Fontaine, D. Savery and G. Cloutier, "Simulation of ultrasound backscattering by red blood cell aggregates: effect of shear rate and anisotropy, Biophysical Journal, vol. 82, pp. 1696-1710, 2002.

[14] I. Fontaine, M. Bertrand and G. Cloutier, "A system-based approach to modeling the ultrasound signal backscattered by red blood cells", Biophysical Journal, vol. 77, pp. 2387-2399, 1999.

[15] D. Savery and G. Cloutier, "Effect of Red Cell Clustering and Anisotropy on Ultrasound Blood Backscatter: A Monte Carlo Study", IEEE Trans. Ultras. Ferroelectr. Freq. Control., vol. 52, no. 1, pp. 94-103, 2005.

[16] R. K. Saha, E. Franceschini and G. Cloutier, "Assessment of accuracy of the structure-factor-size-estimator method in determining red blood cell aggregate size from ultrasound spectrum backscattering coefficient”, J. Acoust. Soc. Am., vol. 129, no. 4, pp. $2269-2277,2011$.

[17] I. Fontaine and G. Cloutier, "Modeling the frequency dependence (5-120 MHz) of ultrasound backscattering by red cell aggregates in shear flow at a normal hematocrit", J. Acoust. Soc. Amer., vol. 113, no. 5, pp. 2893-2900, 2003.

[18] F. T. H. Yu and G. Cloutier, "Experimental ultrasound characterization of red blood cell aggregation using the structure factor size estimator", J. Acoust. Soc. Am., vol. 122, no. 1, pp. 645-656, 2007.

[19] F. T. H. Yu, E. Franceschini, B. Chayer, J. K. Armstrong, H. J. Meiselman and G. Cloutier, "Ultrasonic parametric imaging of erythrocyte aggregation using the structure factor size estimator", Biorheology, vol. 46, pp. 343363, 2009.

[20] G. T. Kuster and M. N. Toksoz, "Velocity and attenuation of seismic waves in two-phase media: part I. Theoretical formulations", Geophysics, vol. 39, no. 5, pp. 587-606, 1974

[21] D. Savery and G. Cloutier, "High-frequency ultrasound backscattering by blood: Analytical and semi-analytical models of the erythrocyte cross section”, J. Acoust. Soc. Amer., vol. 23, no. 4, pp. 3963-3971, 2007.

[22] M. F. Insana and D. G. Brown, "Acoustic scattering theory applied to soft biological tissues", in Ultrasonic Scattering in Biological Tissues, edited by K. K. Shung and G. A. Thieme (CRC, Boca Raton, FL, 1993), Chap.4, pp. 76-124.

[23] R. D. Doolittle and H. Uberall, "Sound scattering by elastic cylindrical shells", J. Acoust. Soc. Amer., vol. 39, no. 2, pp. 272-275, 1965. 
[24] V. P. Dick and A. P. Ivanov, "Extinction of light in dispersive media with high particle concentrations: applicability limits of the interference approximation", J. Opt. Soc. Am., vol. 16, pp. 1034-1039, 1999.

[25] F. L. Lizzi, M. Ostromogilsky, E. J. Feleppa, M. C. Rorke, and M. M. Yaremko, "Relationship of ultrasonic spectral parameters to features of tissue microstructure", IEEE Trans. Ultrason. Ferroelect. Freq. Contr., vol. 33, pp. 319-329, 1986.

[26] B. Lim and R. S. C. Cobbold, "On the relation between aggregation, packing and the backscattered ultrasound signal for whole blood", Ultrasound in Med. Biol., vol. 25, No. 9, pp. 13951405, 1999

[27] T. E. Doyle, A. T. Tew, K. H. Warnick and B. L. Carruth, "Simulation of elastic wave scattering in cells and tissues at the microscopic level”, J. Acoust. Soc. Am., vol. 125, pp. 1751-1767, 2009.

[28] W. S. Jodrey and E. M. Tory, "Computer simulation of close random packing of equal spheres, Physical Review A, vol. 32, pp. 2347-2351, 1985.

[29] X. Weng, G. Cloutier, R. Beaulieu, G. O. Roederer, "Influence of acute-phase proteins on erythrocyte aggregation", Am. J. Physiol., vol. 271, pp. H2346-H2352, 1996.

[30] H. Schmid-Schnbein, H. Malotta and F. Striesow, "Erythrocyte aggregation: causes, consequences and methods of assessment", Tijdschr NVKS, vol. 15, pp. 88-97, 1990.

[31] H. Schmid-Schnbein, G. Gallasch, J. V. Gosen, E. Volger and H. J. Klose, "Red cell aggregation in blood flow. I. New methods of quantification”, Klin. Wschr., vol. 54, pp. 149-157, 1976.

[32] F. T. H. Yu, J. K. Armstrong, J. Tripette, H. J. Meiselman and G. Cloutier, "A local increase in red blood cell aggregation can trigger deep vein thrombosis: Evidence based on quantitative cellular ultrasound imaging", J. Thrombosis and Haemostasis, vol. 9, pp. 481-488, 2011.

[33] E. Franceschini E., F. T. H. Yu, F. Destrempes and G. Cloutier, "Ultrasound characterization of red blood cell aggregation with intervening attenuating tissue-mimicking phantoms using the structure factor size and attenuation estimator”, J. Acoust. Soc. Am., vol. 127, pp. 11041115, 2010.

[34] R. M. Vlad, R. K. Saha, N. M. Alajez, S. Ranieari, G. J. Czarnota and M. C. Kolios, "An increase in cellular size variance contributes to the increase in ultrasound backscatter during cell death", Ultrasound in Medicine \& Biology, vol. 9, pp. 1546-1558, 2010. 


\section{TABLE CAPTION}

Table I: Acoustical properties of blood found in [11] and [21].

TABLE I

\begin{tabular}{lccc}
\hline & $\begin{array}{c}\text { Density } \\
\rho\left(\mathrm{kg} . \mathrm{m}^{-3}\right)\end{array}$ & $\begin{array}{c}\text { Compressibility } \\
\kappa\left(\mathrm{Pa}^{-1}\right)\end{array}$ & $\begin{array}{c}\text { Impedance } \\
Z \text { (MRayl) }\end{array}$ \\
\hline RBC & 1092 & $3.41 \times 10^{-10}$ & 1.766 \\
\hline Plasma & 1021 & $4.09 \times 10^{-10}$ & 1.580 \\
\hline
\end{tabular}




\section{FIGURE CAPTIONS}

Figure 1. Schematic representation of aggregates treated as individual scatterers. The aggregates of RBCs in blood (left side) are assumed to be homogeneous particles (right side) with effective properties that depends on the internal hematocrit, and density and compressibility of the RBCs within them.

Figure 2. Distributions of aggregated RBCs for a constant aggregated radius $r_{a g}=6.32 a=17.32 \mu \mathrm{m}$ and a constant systemic hematocrit $\phi=20 \%$ at three internal hematocrits: a) $\phi_{i}=40 \%$, b) $\phi_{i}=50 \%$ and c) $\phi_{i}=60 \%$. The displayed areas are $300 \mu m$ by $300 \mu m$ in order to enhance the RBC visualization.

Figure 3. (a) and (b): Dependence of the backscattering coefficients on the internal hematocrit: (a) $r_{a g} / a=6.32$ and $\phi=10 \%$, and (b) $r_{a g} / a=6.32$ and $\phi=20 \%$. The symbols represent the $B S C$ computation with the SFM and the dashed lines the $B S C_{e q}$ computation with the EMTSFM. (c) and (d): Corresponding relative errors for the $B S C_{e q}$ versus frequency.

Figure 4. Spectral slope in the frequency bandwidth $5-15 \mathrm{MHz}$ as a function of the internal hematocrit at different systemic hematocrits, the aggregate size being fixed $\left(r_{a g} / a=6.32\right)$. The solid lines represent the SSs for the $B S C$ computed with the SFM and the dashed lines the SSs for the $B S C_{e q}$ computed with the EMTSFM.

Figure 5. Dependence of the backscattering coefficients for different aggregate sizes and a constant internal hematocrit $\phi_{i}=60 \%$ at systemic hematocrits of 10,20 and $30 \%$. The symbols represent the BSC computation with the SFM and the dashed lines the $B S C_{e q}$ computation with the EMTSFM.

Figure 6. Dependence of the backscattering coefficients for different aggregate sizes and a constant internal hematocrit $\phi_{i}=60 \%$ at systemic hematocrits of 10,20 and 30\%. The symbols represent the BSC computation with the SFM and the dashed lines the $B S C_{e q_{L F}}$ computation with the EMTSFM using the Percus-Yevick approximation.

Figure 7. (a) Frequency limit $f_{l}$ for which the relative error of $B S C_{e q}$ was inferior to $30 \%$ as a function of the aggregate size studied. The solid lines are for the EMTSFM and the dashed lines for the low frequency approximation of the EMTSFM. (b) Corresponding product $k_{l} r_{a g}$.

Figure 8. Spectral slope in the frequency bandwidth $5-15 \mathrm{MHz}$ as a function of the aggregate size at different systemic hematocrits, the internal hematocrit being fixed ( $\phi_{i}=60 \%$ for aggregated RBCs). The solid lines represent the SSs for the $B S C$ computed with the SFM and the dashed lines the SSs for the $B S C_{e q}$ computed with the EMTSFM.

Figure 9. (a) Structure factors $S$ versus frequency used in the computation of the SFM for a fixed systemic hematocrit $\phi=30 \%$, a fixed internal hematocrit $\phi_{i}=60 \%$ and three aggregate radii $r_{a g} / a=3.16,5.0$ and 7.07. (b) Structure factors $S_{a g}$ versus frequency used in the computation of the EMTSFM for the same aggregating conditions. 


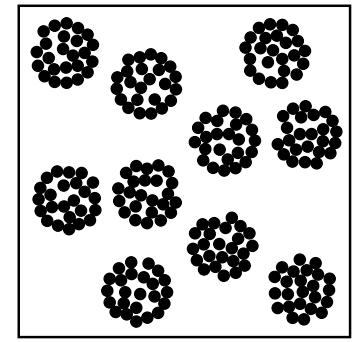

Reality

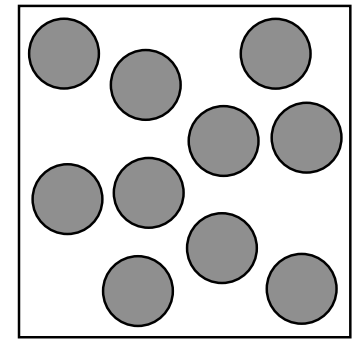

Effective medium approximation

Fig. 1. 


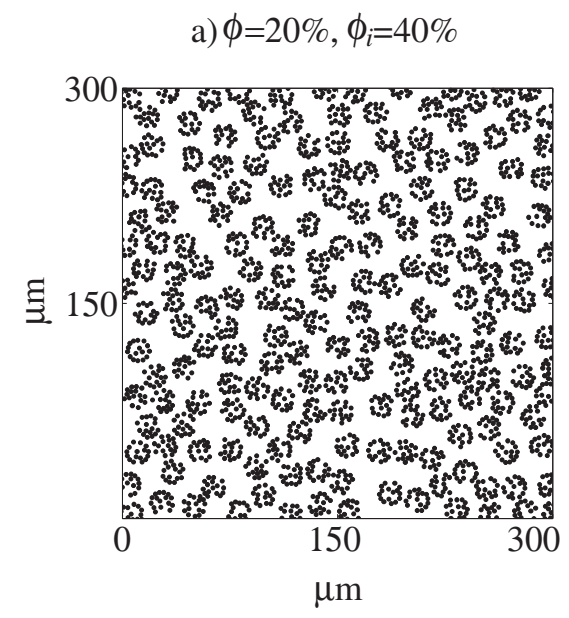

b) $\phi=20 \%, \phi_{i}=50 \%$

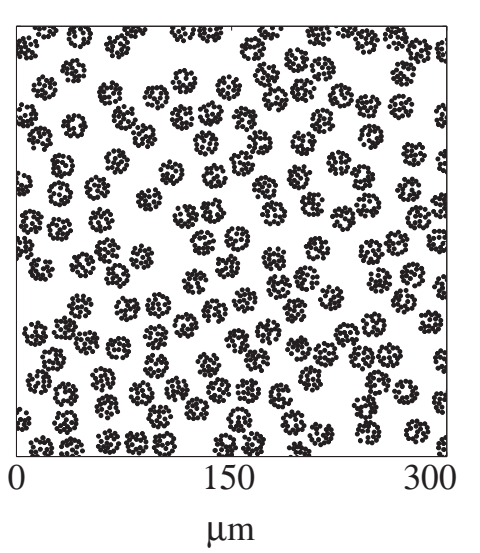

c) $\phi=20 \%, \phi_{i}=60 \%$

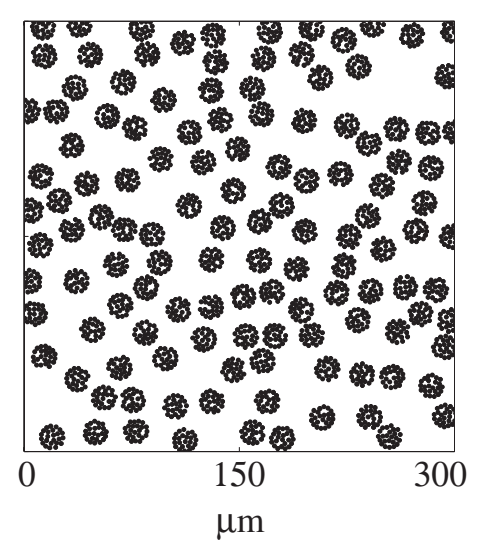

Fig. 2. 

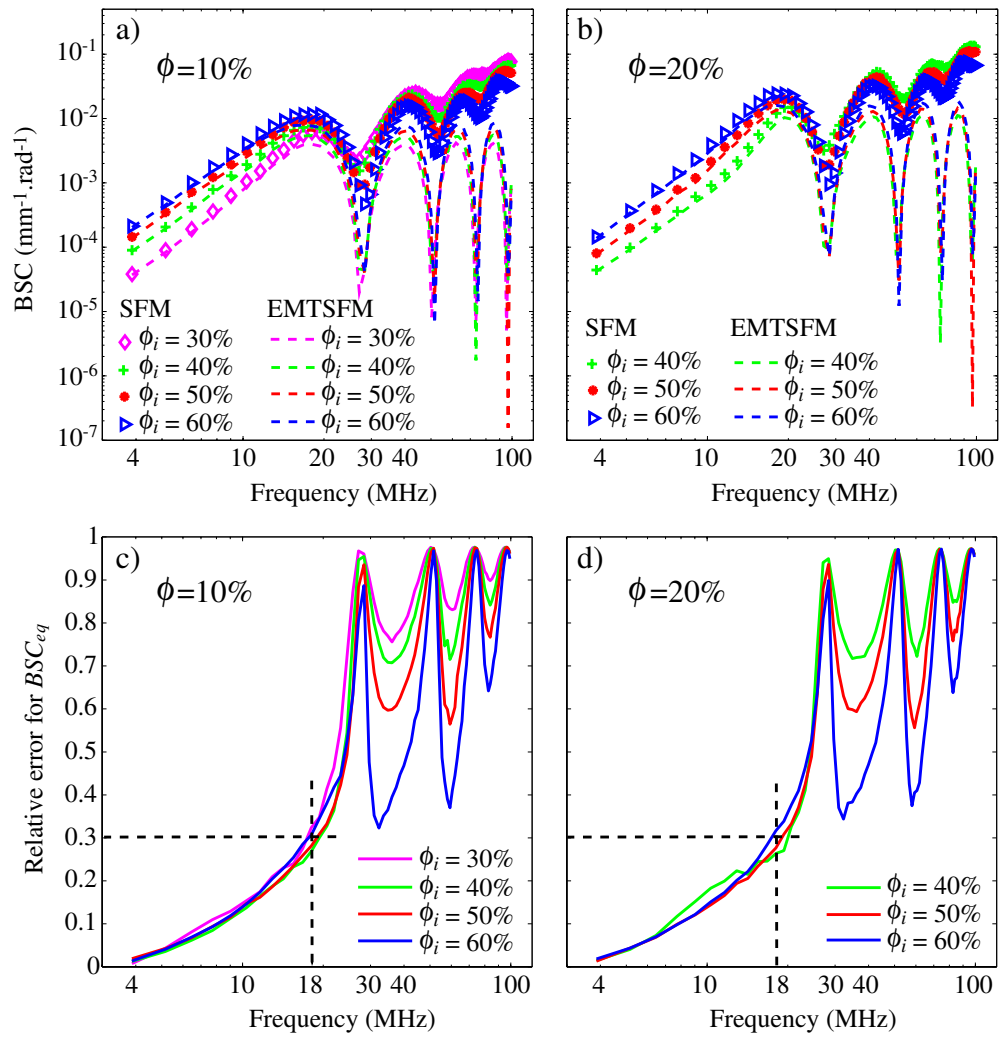

Fig. 3. 


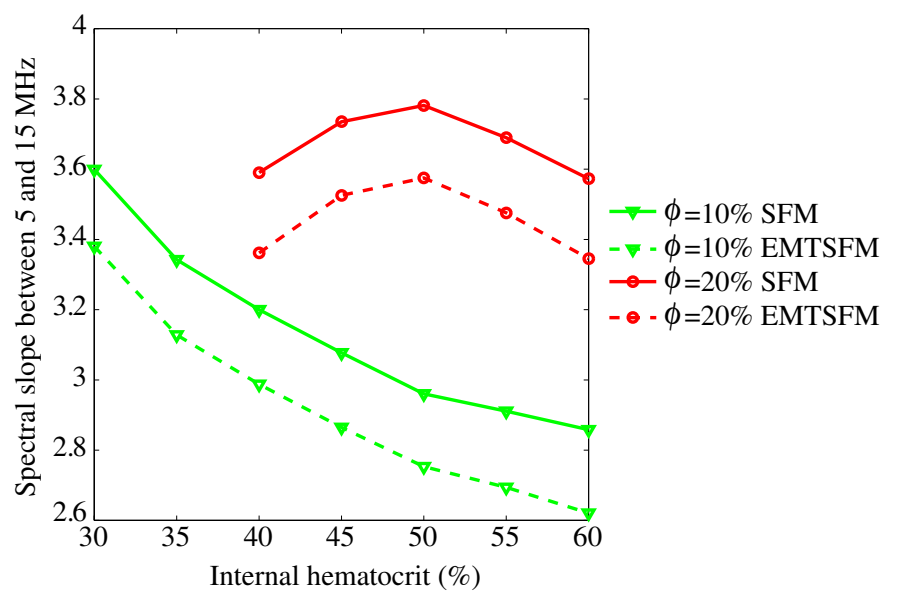

Fig. 4. 

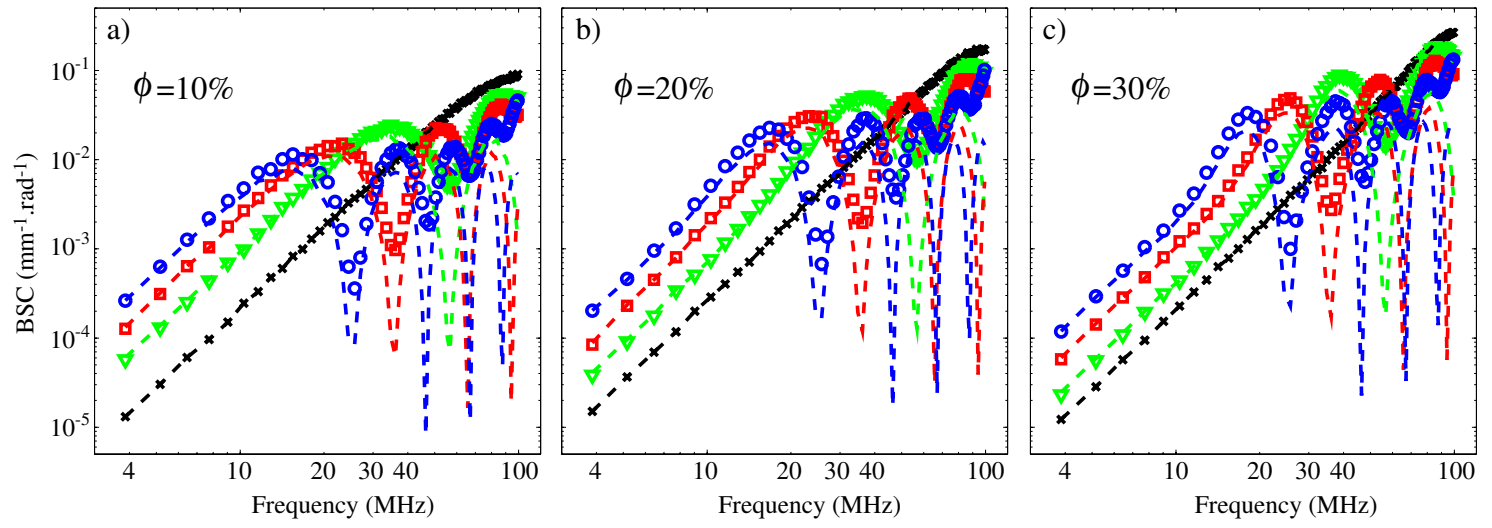

SFM

* $r_{a g} / a=1$

$\nabla r_{a g} / a=3.16$

- $r_{a g} a=5$

- $r_{a g} / a=7.07$

EMTSFM

$-r_{a g} / a=1$
$---r_{a g} / a=3.16$

- - $r_{a g} / a=5$

- - $r_{a g} / a=7.07$

Fig. 5. 

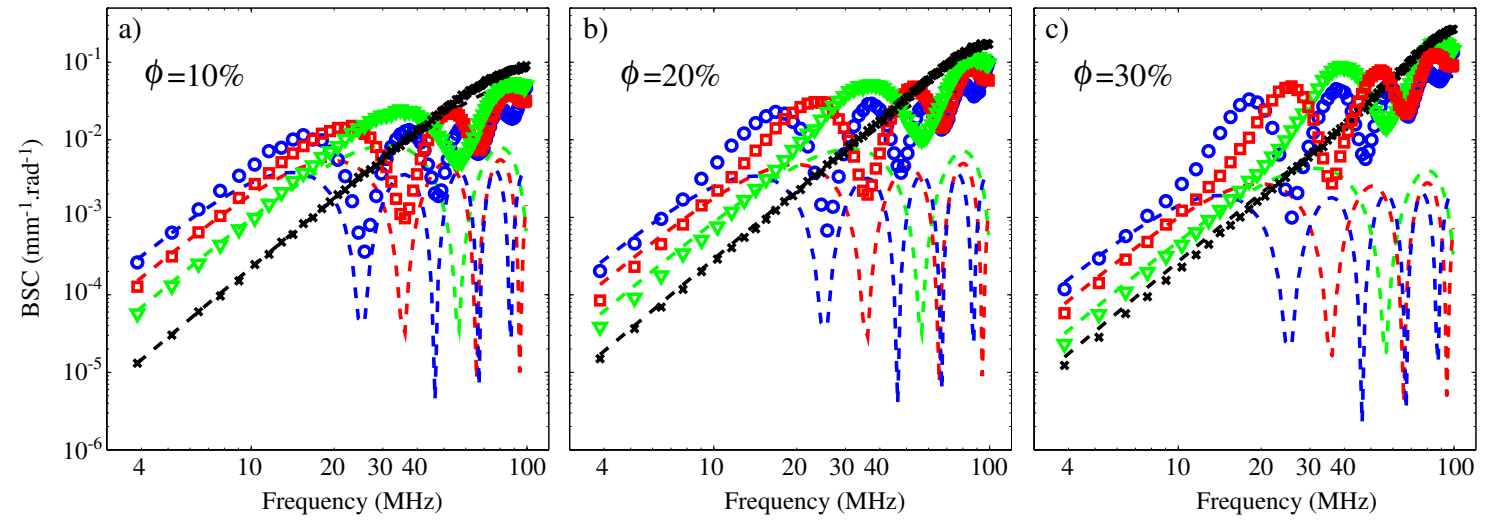

SFM

* $r_{a g} / a=1$

$\nabla r_{a g} / a=3.16$

- $r_{a g} / a=5$

- $r_{a g} / a=7.07$

EMTSFM (LF)

- - $r_{a g} / a=1$

$--r_{a g} / a=3.16$

$\begin{array}{ll}--- & r_{a g} / a=5 \\ --- & r_{a g} / a=7.07\end{array}$

Fig. 6. 

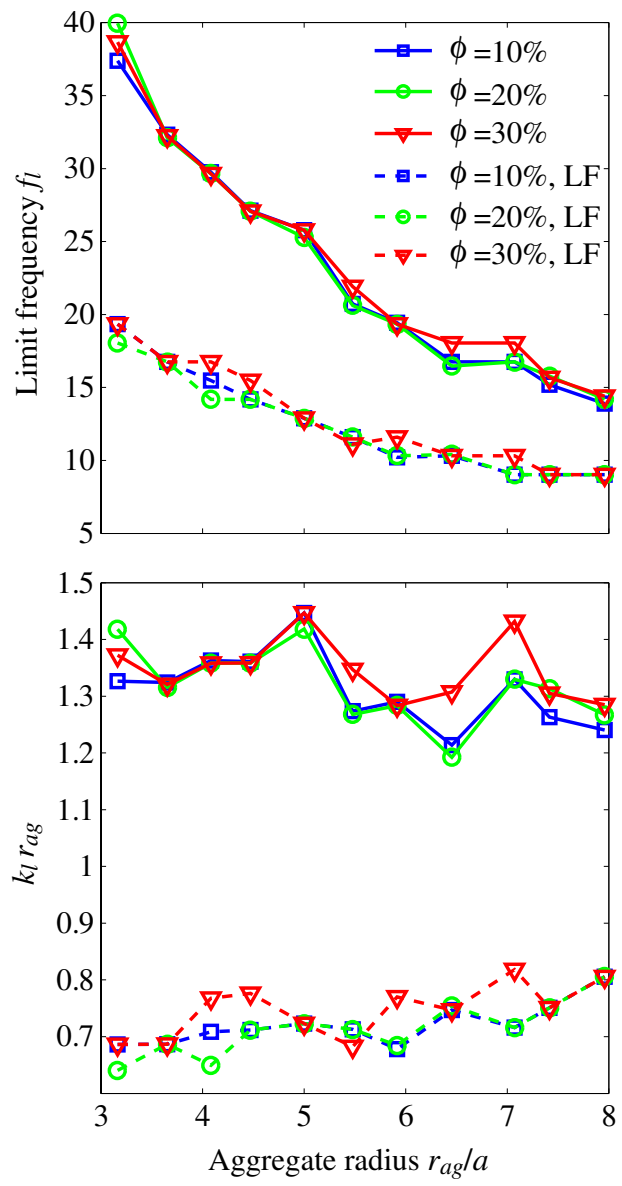

Fig. 7. 


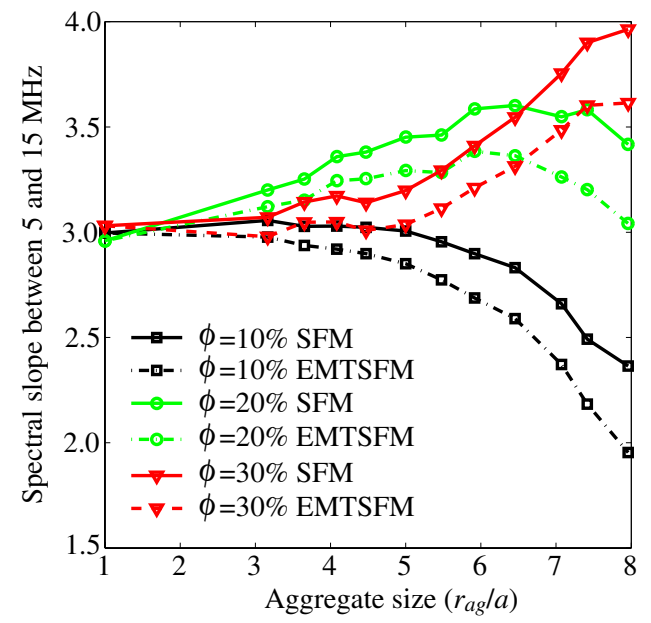

Fig. 8 . 

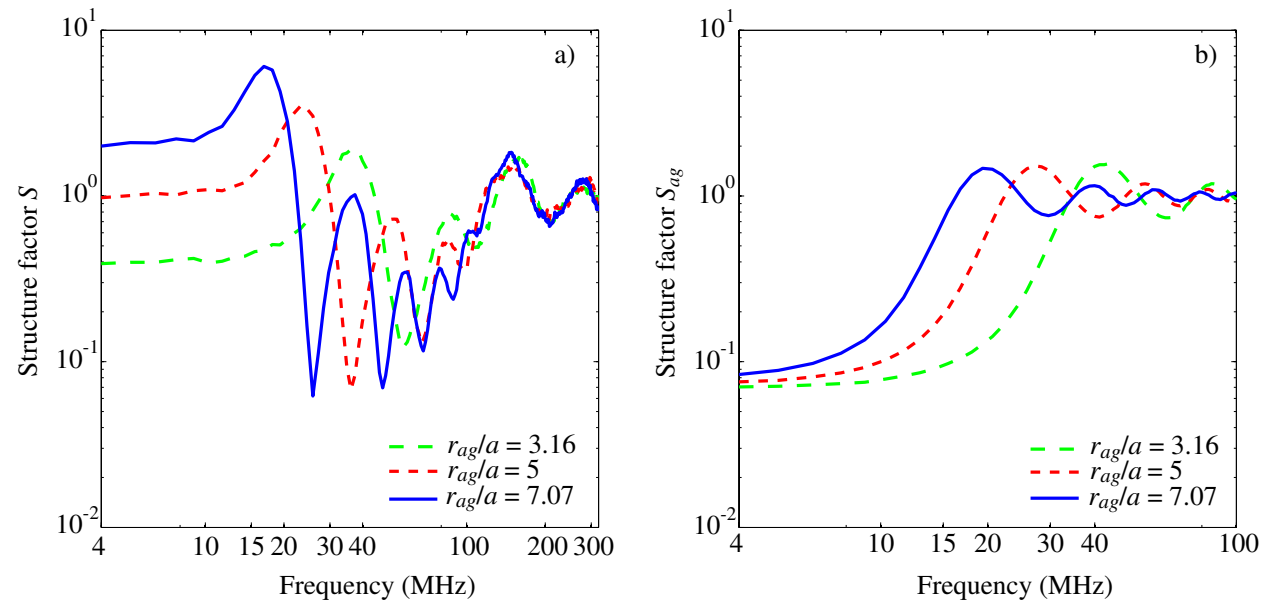

Fig. 9. 